Classification

Physics Abstracts

$42.30 \mathrm{va}$

\title{
Interférometrie haute résolution par microscopie optique en champ proche
}

\author{
Fadi Baida, Claudine Bainier et Daniel Courjon
}

Laboratoire d'Optique P. M. Duffieux, CNRS URA 214, Université de Franche-Comté, Faculté des Sciences et des Techniques, Route de Gray, 25030 Besançon Cedex, France

(Reçu le 4 juillet; accepté le 26 octobre, 1994)

\begin{abstract}
Résumé . - Linterférométrie optique en champ proche est basée sur l'exploitation des interférences entre les ondes évanescentes créées lors de la diffraction de la lumière par les détails de l'objet dont la taille est inférieure à la demi-longueur d'onde du rayonnement utilisé. Dans cette communication nous présentons une étude expérimentale de réalisation et de détection de ces interférences ainsi qu'une approche théorique simple permettant de décrire cette expérience.
\end{abstract}

Abstract . - Near Field Optical Interferometry is based on the exploitation of interferences between evanescent fields generated by diffraction of light on subwavelength object features. In this communication, we present a technique allowing to detect such interference patterns. Parallely, a simple theoretical approach is developed.

\section{Introduction.}

Linterférométrie optique classique résultant de l'interaction de deux ou de plusieurs faisceaux présentant un certain état de cohérence, est l'un des phénomènes les plus utilisés en optique pour réaliser des hologrammes, des opérations de filtrage et pour mettre en oeuvre des techniques de mesures dimensionnelles dans la gamme micrométrique et submicrométrique. Cette interférométrie met en contribution les ondes homogènes (progressives) de la lumière caractérisées par un vecteur d'onde à composantes réelles.

Pour décrire ce phénomène, considérons deux ondes planes monochromatiques de même amplitude se propageant dans un milieu d'indice $n$ et faisant un angle $2 \theta$ entre elles (Fig. 1a), dans la zone où ces deux faisceaux se croisent, le champ électrique s'écrit :

$$
E=\exp \left(j \omega t-j \mathbf{k}_{1} \mathbf{r}\right)+\exp \left(j \omega t-j \mathbf{k}_{2} \mathbf{r}\right)
$$

Les vecteurs $\mathbf{k}_{1}$ et $\mathbf{k}_{2}$ ont respectivement les composantes : $k_{1 x}=k \cos \theta, k_{1 y}=k \sin \theta, k_{2 x}=k$ $\cos \theta$ et $k_{2 y}=-k \sin \theta\left(k=\frac{2 \pi n}{\lambda}\right)$.

Lintensité lumineuse s'écrit alors :

$I(x, y, z)=|E|^{2}=|\exp (j \omega t-j k \cos \theta x)\{\exp (-j k \sin \theta y)+\exp (j k \sin \theta y)\}|^{2}=\frac{1}{4} \cos ^{2}(k \sin \theta y)$ 
Celle-ci varie donc sinusoïdalement suivant l'axe $y$ et admet un interfrange (distance entre deux franges) de $\frac{\lambda}{2 n \sin \theta}$. Pour que ce système de franges soit utilisable comme "porteuse" et que la quantité d'information codée soit maximale, il faut que sa fréquence soit la plus grande possible c'est à dire telle que le rapport $\frac{\lambda}{2 n \sin \theta}$ soit minimal. Cette limite imposée sur la fréquence du système de franges n'est autre que celle du critère de Rayleigh appliquée en imagerie optique. Il stipule que la composante latérale du vecteur d'onde est toujours limitée à $k \sin \theta$.

La meilleure solution revient alors à travailler avec deux faisceaux se propageant dans deux directions opposées ( $\sin \theta=1$ ) et dans un milieu d'indice nettement supérieur à celui de l'air (Fig. 1b). De telles conditions sont expérimentalement difficiles à assurer.
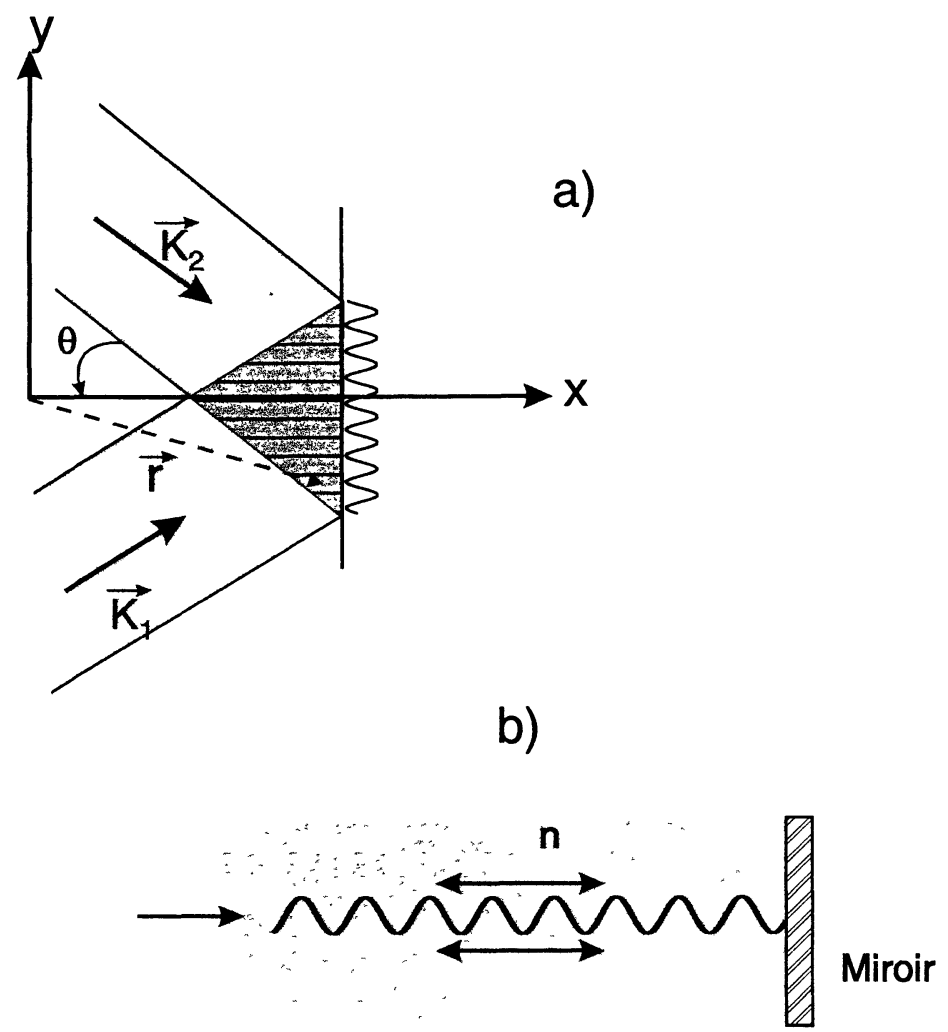

Fig. 1. - a) Deux ondes planes interfèrent en donnant lieu à un système de franges sinusoïdales d'interfrange $\frac{\lambda}{2 n \sin \theta}$. b) Pour diminuer cette valeur, le milieu doit avoir un indice supérieur à 1 et les deux faisceaux doivent se propager en sens opposés.

[a) Interference pattern is generated by two plane waves, the period is equal to $\frac{\lambda}{2 n \sin \theta}$. b) To decrease this value, we must work in a medium which optical index is greater than 1 and the two waves have to propagate in opposite direction.]

Considérons maintenant des ondes évanescentes créées lors de la diffraction de la lumière par des structures de l'objet de dimensions sublongueur d'onde. Ces ondes, dites non homogènes, se propagent parallèlement à la surface moyenne de l'objet et leur amplitude décroît exponentiellement quand on s'éloigne de ce dernier. Cette décroissance exponentielle implique que la composante $k_{z}$ du vecteur d'onde soit imaginaire pure. Sachant que $k^{2}=k_{x}^{2}+k_{y}^{2}+k_{z}^{2}$, il s'en suit 


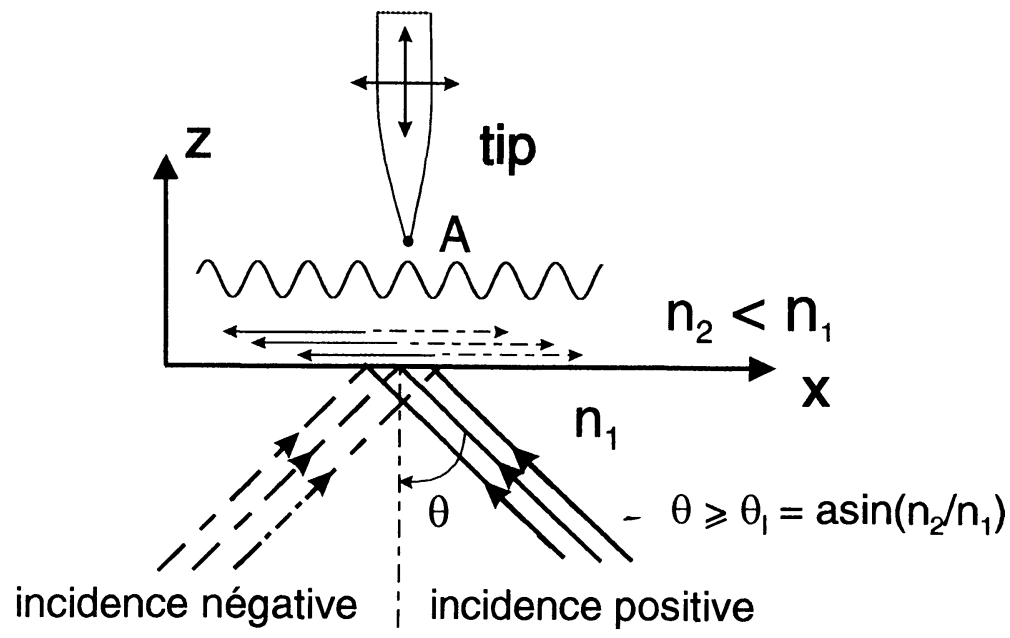

Fig. 2. - Montage en réflexion totale utilisant plusieurs ondes. Les ondes évanescentes ainsi créées interfèrent entre elles. Il en résulte un système de franges exactement comme dans le cas classique d'un interféromètre de Fabry-Pérot $\left(\theta_{l}\right.$ est l'angle limite de réfraction).

[Total internal reflection device using multiple waves. The resulting evanescent waves will interfere in order to generate a similar interference pattern as in the classical case of a Fabry-Pérot interferometer $\left(\theta_{l}\right.$ is the refraction limit angle).]

que $\left|k_{x}\right|$ et/ou $\left|k_{y}\right|$ peuvent prendre des valeurs très supérieures à $|k|$. Pour ces ondes particulières, la limite de Rayleigh pourra être dépassée.

En conséquence, comme pour les ondes classiques, les ondes évanescentes pourront interférer entre elles pour donner lieu à un système de franges stationnaires d'interfrange plus petite que $\frac{\lambda}{2 n \sin \theta}$. Les premières expériences visant à vérifier l'existence de telles interférences ont été effectuées en 1968 et 1969 [1, 2]. La détection en champ proche n'existant pas à l'époque, la mise en évidence de ce phénomène a été effectuée par voie holographique.

Deux équipes européennes $[3,4]$ ont récemment effectué une mesure directe de ces interférences par microscopie optique en champ proche. Dans les deux cas, il s'agissait d'interférences à deux ondes détectées par deux types de sondes différentes (fibre optique taillée en pointe pour la première et une pointe de microscope à force atomique en nitrure de silicium pour la deuxième).

Dans le cadre de ce travail, nous nous sommes intéressés à l'interférométrie optique à ondes multiples en champ proche afin de tirer bénéfice à la fois des atouts de la microscopie haute résolution en champ proche et des atouts de l'interférométrie optique.

Une des méthodes permettant la mise en évidence d'un tel système consiste à placer un microscope optique à effet tunnel (travaillant en réflexion totale) à l'intérieur d'un interféromètre de type Fabry-Pérot [5]. La structure de la distribution lumineuse au-dessus de l'objet permet de vérifier l'existence de ces ondes non homogènes stationnaires. Dans cette communication, nous effectuerons une étude expérimentale de ce phénomène. Une partie sera consacrée à l'influence des paramètres tels que la polarisation et la distance d'analyse sur la valeur de l'intensité lumineuse détectée.

\section{Principe et montage expérimental.}

Pour des raisons de simplicité, nous avons choisi d'utiliser les ondes évanescentes de Fresnel (plus facile à maîtriser que la diffraction) créées par réflexion totale de la lumière sur un dioptre séparant deux milieux d'indices différents (Fig. 2). Chaque rayon tombant sous une incidence 
positive va générer une onde évanescente se propageant dans la direction de la composante tangentielle du vecteur d'onde. De même, les rayons arrivant sous une incidence négative vont donner lieu à des ondes se propageant dans le sens opposé et ces ondes vont interférer entre elles pour constituer un système de franges stationnaires. La détection de ce système de franges a lieu au moyen d'une sonde locale (fibre optique taillée en pointe).

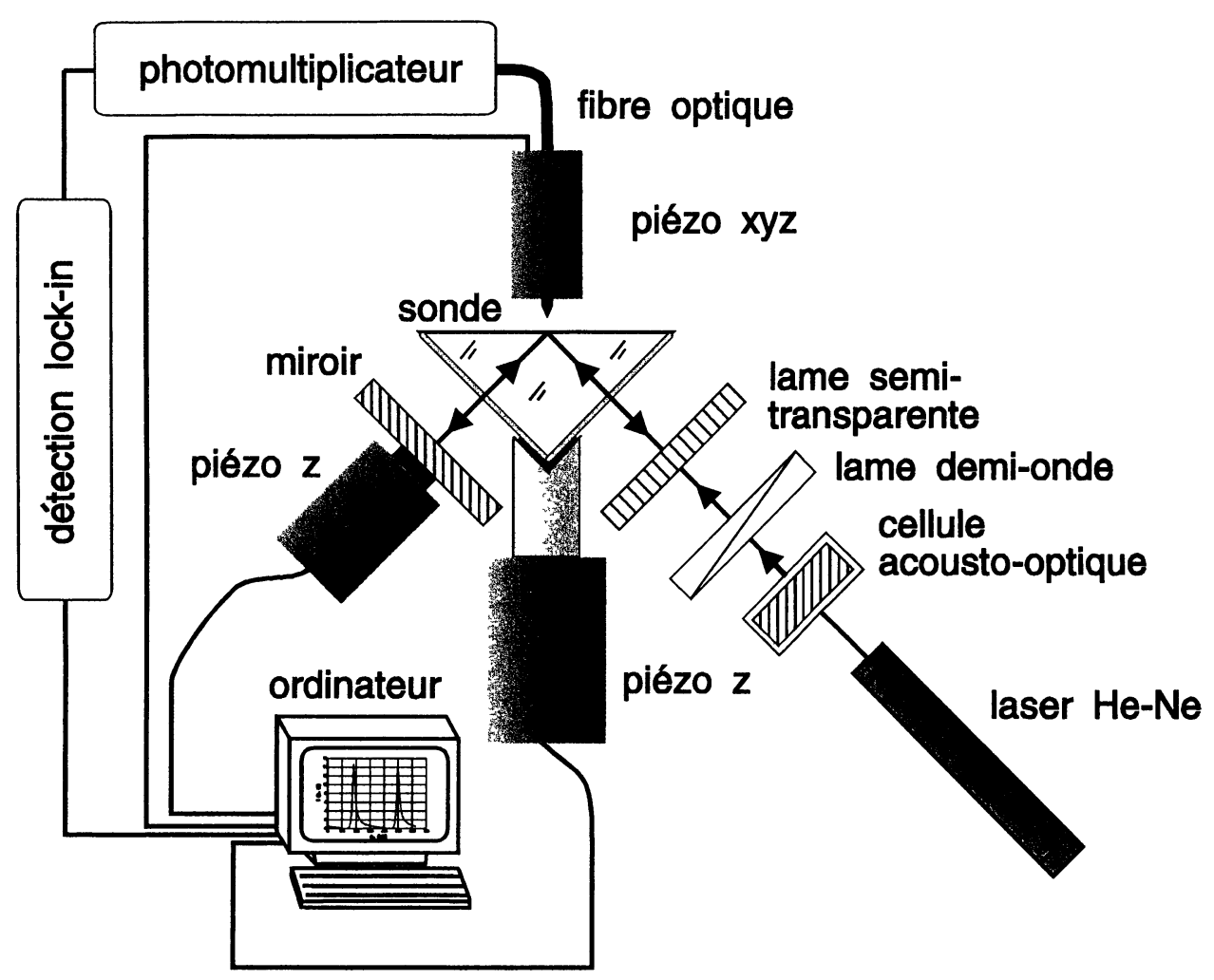

Fig. 3. - Schéma du montage expérimental.

[Schematic of the experimental set-up.]

Le montage expérimental est représenté sur la figure 3. Un interféromètre Fabry-Pérot constitué d'un miroir monté sur un translateur piézo-électrique et d'une lame semi-transparente est utilisé pour générer le système de franges à la surface du prisme à réflexion totale. Le microscope, de type STOM [6] (Scanning Tunneling Optical Microscope), est placé à l'intérieur de l'interféromètre de telle sorte que la lumière réfléchie par l'objet soit renvoyée sur ce dernier par le miroir. La sonde à effet tunnel est une fibre optique monomode étirée à chaud puis attaquée chimiquement à l'acide fluorhydrique pour réduire au minimum la dimension de son extrémité. Cette sonde est placée au voisinage de l'objet à l'aide d'un tube piézo-électrique afin que les ondes évanescentes dues aux multiples réflexions totalés puissent être diffractées par la sonde et ainsi converties en ondes homogènes détectables à grande distance. Un photomultiplicateur (PM), refroidi à $-18^{\circ} \mathrm{C}$, est utilisé pour amplifier la détection de ces ondes. Le faisceau incident est issu d'un laser He-Ne modulé en amplitude ; une détection synchrone est alors effectuée sur le signal détecté par l'intermédiaire d'un amplificateur lock-in. Le balayage de l'objet par la fibre est piloté au moyen d'un ordinateur de type IBM 386. Le prisme utilisé est un prisme à réflexion 
totale en BK7 dont les deux faces ont subi un traitement antireflet afin de limiter les réflexions parasites. Le miroir $M$ peut être déplacé parallèlement à lui-même à l'aide d'un translateur piézoélectrique parfaitement corrigé de l'hystérésis. Les coefficients de réflexion en énergie de la lame semi-transparente et du miroir sont de $70 \%$ et $92 \%$ respectivement.

\section{Théorie.}

Lidée générale consiste à calculer l'intensité lumineuse en un point quelconque situé dans le milieu de sortie. Lobjet (surface du prisme) et la sonde sont caractérisés par un coefficient de transmission $t$ et un coefficient de réflexion $r$ (en amplitude).

Le calcul est basé sur la résolution des équations de Maxwell avec les conditions de continuité imposées sur les champs électrique et magnétique.

Le schéma de principe est présenté par la figure 4. Le champ incident est une onde plane monochromatique polarisée rectilignement, d'amplitude $E_{0}$. Soient $r_{1}$ et $r_{2}$ les coefficients de réflexion en amplitude de la lame semi-transparente et du miroir respectivement et $t_{1}$ le coefficient de transmission (en amplitude aussi) de la lame semi-transparente.



Fig. 4. - Schéma de principe de l'interféromètre à ondes multiples réalisé. Lépaisseur de la cavité peut être modifiée en changeant la position de la lame semi-transparente, $e_{1}$, suivant $\mathbf{u}$ ou celle du miroir, $e_{2}$, suivant $\nu$. La sonde, en balayant la surface du prisme, se comporte comme un détecteur se déplaçant à l'intérieur de la cavité interférométrique.

[Principle of the multiple wave interferometer. The length of the cavity can be modified by moving the beamsplitter, $e_{1}$, in the $\mathbf{u}$ direction, or by moving the mirror, $e_{2}$, in the $\nu$ direction. By scanning the prism surface, the tip is playing the role of a detector moving inside the interferometric cavity.] 
Le champ lumineux calculé à la distance $z$ de l'objet prend alors la forme :

$$
\begin{aligned}
& E(x, y, z)= \\
& \frac{E_{0} t_{1} t \exp \left(-j \phi_{1}\right)\left[\exp \left\{j\left(K_{x} x+K_{y} y+K_{z} z\right)\right\}+r_{2} r \exp \left\{-j\left(K_{x} x+K_{y} y+K_{z} z+2 \phi_{2}\right)\right\}\right]}{1-r^{2} r_{1} r_{2} \exp \left\{-2 j\left(\phi_{1}+\phi_{2}\right)\right\}}
\end{aligned}
$$

où : $\phi_{1}$ est la phase relative à la distance $e_{1}$ et $\phi_{2}$ est la phase relative à la distance $e_{2} . K_{x}, K_{y}$ et $K_{z}$ sont les composantes de l'un des vecteurs d'onde au-dessus du dioptre.

Dans le cas où la sonde est supposée de taille très petite, le modèle dipolaire (pas de couplage entre la sonde et la lumière) semble être bien adapté. Dans ce cas, $r$ et $t$ sont les coefficients de réflexion et de transmission de Fresnel calculés dans le cas de la réflexion totale, ils ne dépendent que des indices optiques, de l'angle d'incidence et de la polarisation.

Dans le cas où la sonde est large (fibre optique clivée par exemple), ces coefficients $r$ et $t$ sont calculés [7] en modélisant la sonde par un objet plan occupant un demi-espace.

Le cas réel, qui est probablement le cas intermédiaire où la dimension de la sonde lui confère des propriétés dipolaires et de couplage, ne sera pas traité dans ce travail en raison de la complexité et de la lourdeur des calculs nécessaires pour faire intervenir la diffraction de la lumière par une sonde de forme géométrique quelconque (un calcul perturbatif à l'ordre deux est nécessaire pour tenir compte de la diffraction de la lumière dans la détermination des coefficients spéculaires de réflexion et de transmission).

\section{Simulations.}

Les variations de l'intensité (carré du module du champ) le long du dioptre sont présentées sur la figure 5. Dans le plan $(x y)$ l'intensité varie sinusoïdalement lorsqu'on se déplace parallèlement au plan d'incidence et elle est constante dans la direction perpendiculaire.

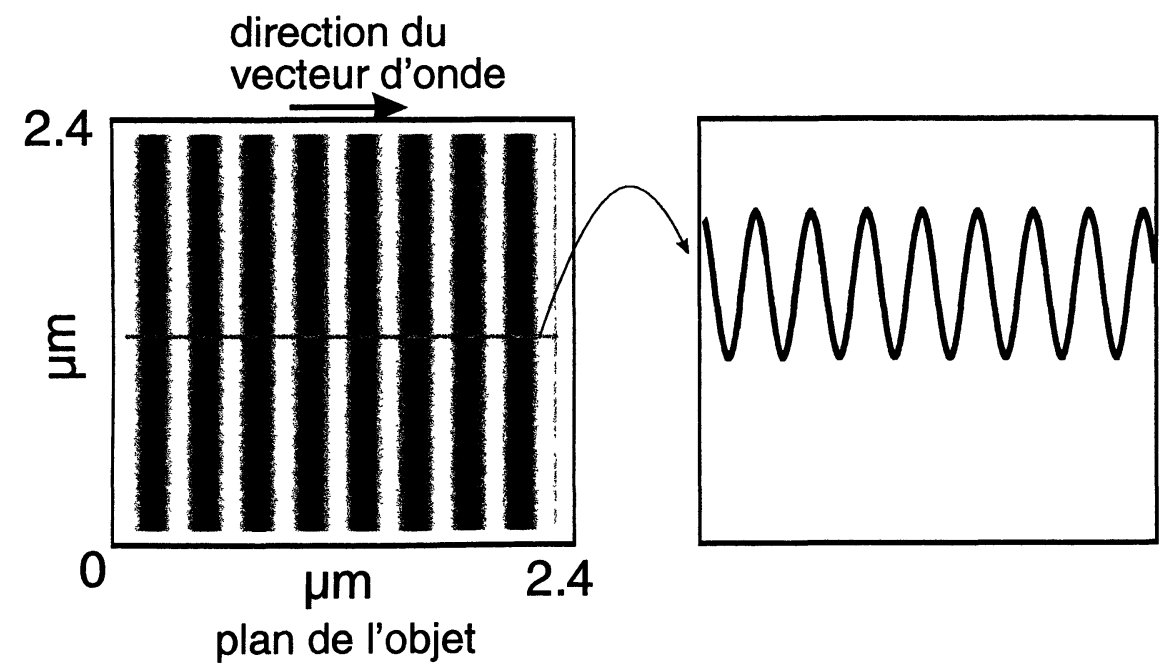

Fig. 5. - Simulation de l'intensité lumineuse détectée à la surface du prisme. Le système de franges est sinusoïdal de période $\frac{\lambda}{2 n \sin \theta}$ et les franges sont perpendiculaires à la composante tangentielle du vecteur d'onde incident.

[Simulation of the distribution of the light intensity on the prism surface. The period is $\frac{\lambda}{2 n \sin \theta}$ and the fringes are perpendicular to the tangential component of the incident wave vector.] 


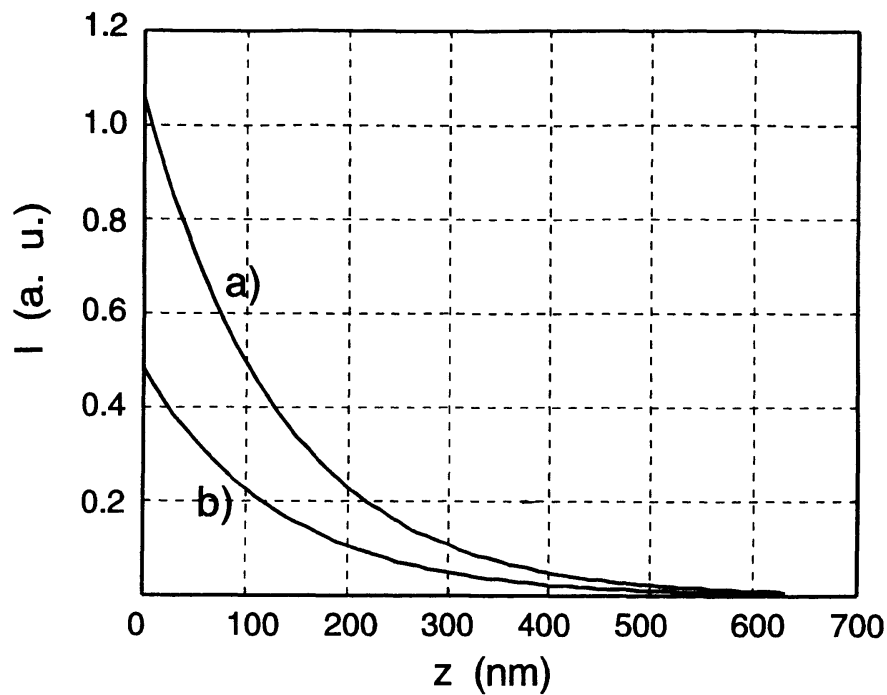

Fig. 6. - Variations de l'intensité lumineuse détectée à l'aide d'une sonde ponctuelle en fonction de la distance qui la sépare de l'objet. a) en TE et b) en TM.

[Simulation of the light intensity variations detected with a punctual tip versus the tip-object distance. a) in TE mode and $b$ ) in TM mode.]

En fonction de la distance $z$ entre la sonde et l'objet et dans le cas d'un modèle dipolaire (sonde fine) les courbes de la figure 6 décroissent exponentiellement (superposition d'une infinité d'exponentielles élémentaires en phase). A un coefficient mụltiplicatif près, ces courbes sont indépendantes de la longueur de la cavité interférométrique.

Il est plus intéressant d'étudier le comportement en $z$ de l'intensité dans le cas d'une sonde plate afin de mettre en évidence l'effet de couplage. Les courbes de la figure 7 traduisent les variations de l'intensité lumineuse détectée par la sonde pour plusieurs positions du miroir. La forme de ces courbes sera discutée à la fin de ce paragraphe. La position de la sonde étant fixée $\left(x_{\mathrm{s}}=0, y_{\mathrm{s}}=0, z_{\mathrm{s}}=1 \mathrm{~nm}\right)$, on trace les variations de l'intensité en fonction de la position du miroir (Fig. 8) en appliquant la méthode dipolaire. La courbe obtenue ressemble à celle d'un interféromètre classique. En effet, notre montage n'est autre qu'un Fabry-Pérot "plié" dont l'observateur (le détecteur) est situé à l'intérieur. Le mouvement du miroir introduit sur toutes les ondes participant à la réflexion totale (sauf la première onde) la même différence de phase. Ceci a pour effet de reproduire assez fidèlement le comportement d'un interféromètre en fonctionnement classique. La dissymétrie observée est liée à la position du détecteur qui est situé à l'intérieur de l'interféromètre détectant ainsi la contribution d'une onde (la première traversant la lame) dont la phase est indépendante de la position du miroir. La courbe est périodique de période $\lambda / 2$. Le coefficient de finesse (largeur à mi-hauteur du pic) de l'interféromètre ne dépend que des coefficients de réflexion et de transmission de la lame et du miroir, il est donc indépendant de la position de la sonde, ce qui implique que la finesse est constante quelle que soit la distance séparant la sonde de la surface.

Ce résultat s'explique par l'absence de couplage entre la pointe supposée très fine et la surface. Considérons maintenant le comportement de l'intensité $I=f\left(e_{2}\right)$ en fonction de la polarisation. Les figures $9 \mathrm{a}$ et $9 \mathrm{~b}$ sont obtenues pour une polarisation TE et TM respectivement. On note que pour les deux polarisations propres TE et TM, le pic de résonance n'a pas lieu pour la même position du miroir. Ce résultat s'explique par la différence du déphasage à la réflexion totale entre 

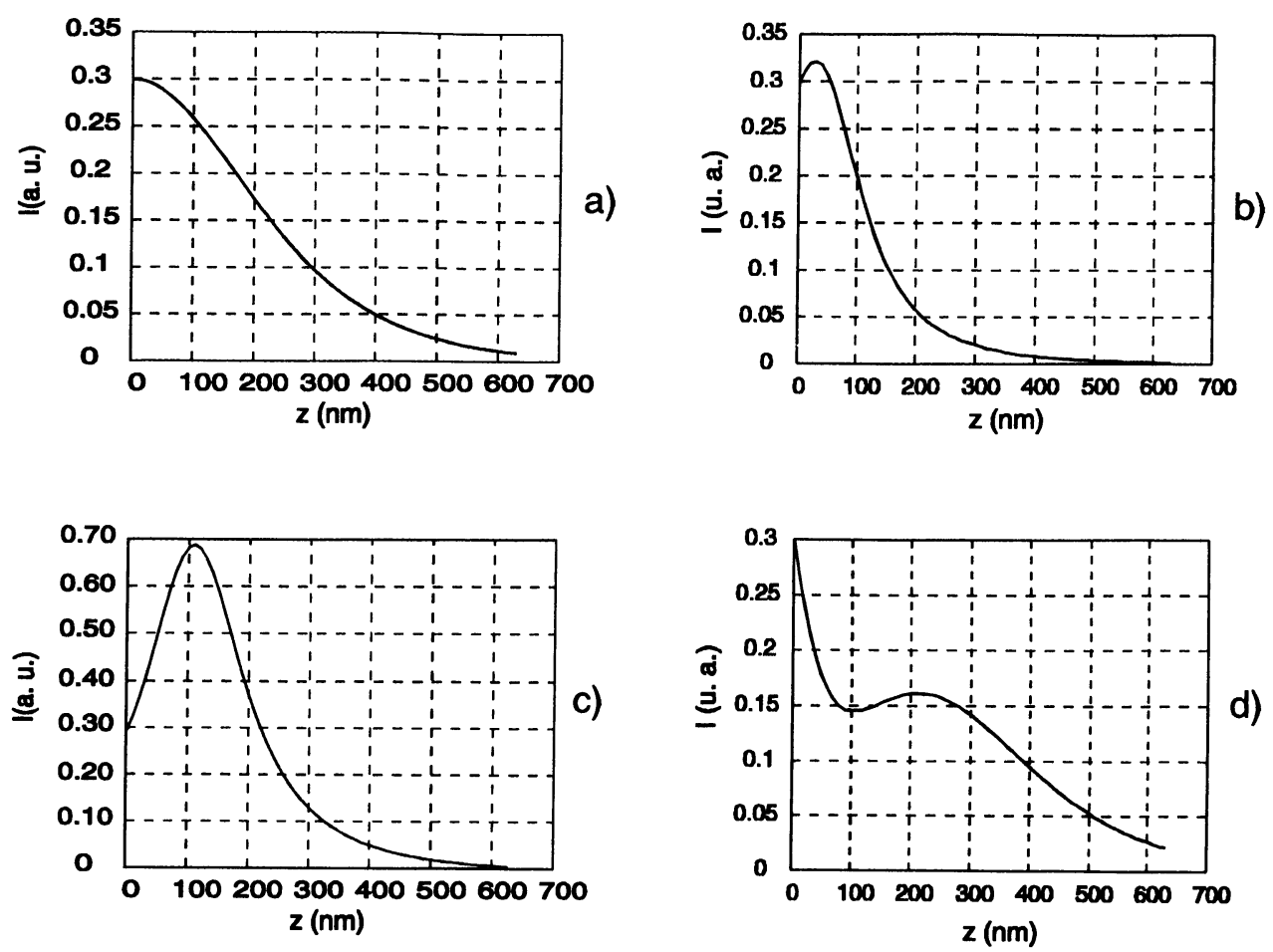

Fig. 7. - Dans le cas d'une sonde plate (fibre clivée), le couplage sonde-objet intervient sur les caractéristiques de la cavité et le comportement en $z$ de l'intensité lumineuse en est affecté. De a) à d) le miroir se déplace par pas de $100 \mathrm{~nm}$.

[In the case of a flat tip (cleaved fiber for example), the coupling between the object and the tip will change the characteristics of the cavity and the behaviour of the intensity versus $z$ is modified (not a simple exponential. From a) to d) the mirror is moving with an increment of $100 \mathrm{~nm}$.]

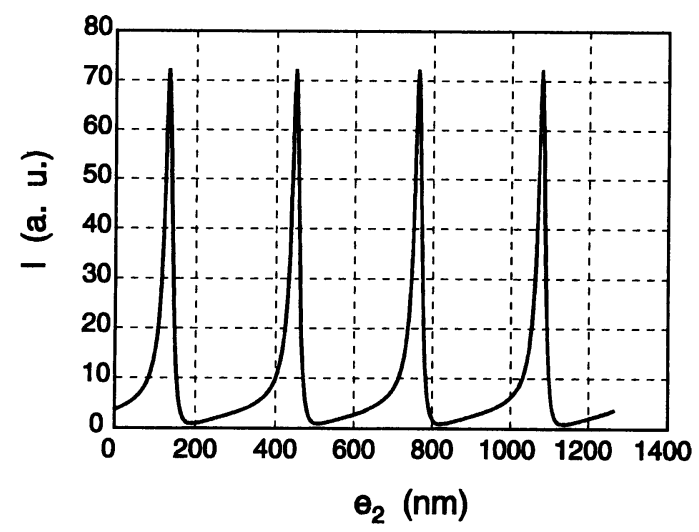

Fig. 8. - Variations de l'intensité lumineuse détectée par la sonde (ponctuelle) en fonction de la position du miroir. La dissymétrie est due à la non équivalence entre les deux bras de l'interféromètre : en effet, pour changer l'épaisseur de la cavité on peut déplacer soit la lame semi-transparente (toutes les ondes subissent le même changement de phase), soit le miroir (toutes les ondes sauf la première vont changer de phase), il y a alors dissymétrie.

[Plotted intensity curves versus the mirror position at a given point of the tip above the prism. According to the parameters, we can note symmetrical or asymmetrical peak shape.] 


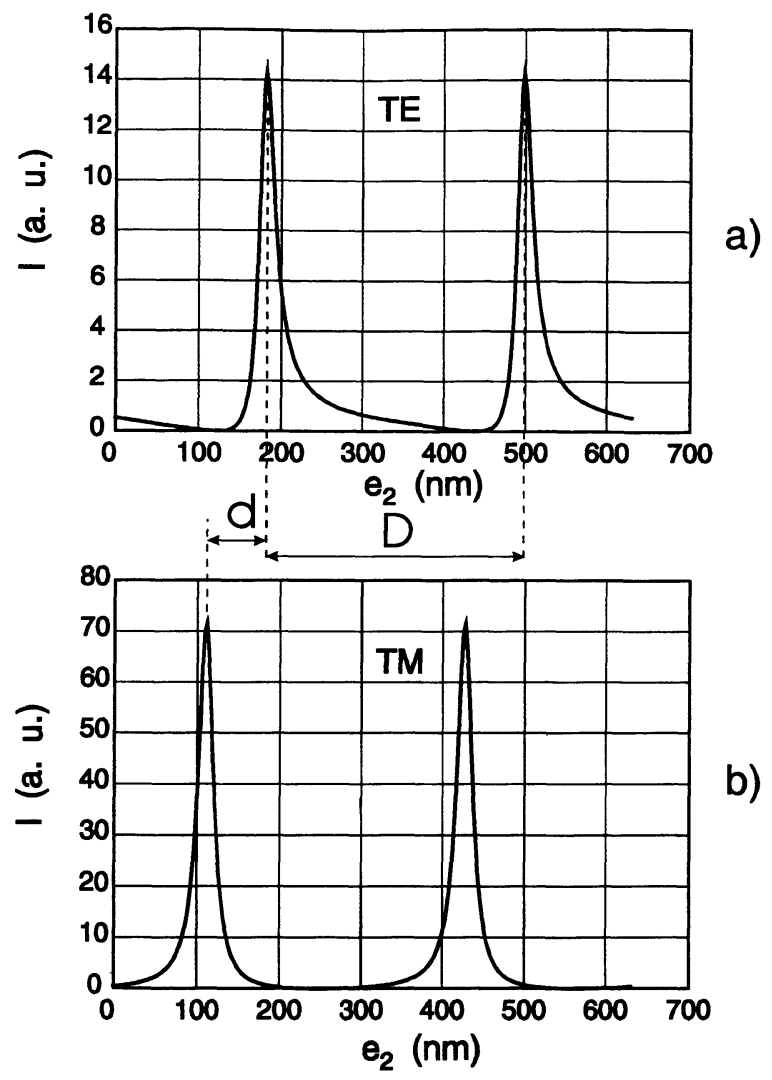

Fig. 9. - Variations de l'intensité détectée en fonction de la position du miroir. a) en TE, b) en TM. La distance entre deux maxima est $D=\frac{\lambda}{2}=\frac{632.8}{2}=316.4 \mathrm{~nm}$ et le glissement des pics, dû au changement de polarisation, vaut $d=69 \mathrm{~nm}$ (il s'agit en fait du glissement de Goos-Hänchen dû à la réflexion totale).

[Plotted intensity curves versus the mirror position. a) and b) correspond to TE and TM modes respectively. The distance between two resonance peaks equals $D=\frac{\lambda}{2}$ and the distance between a TE and a TM peak is $d=69 \mathrm{~nm}$. This phase shift is responsible for the Goos-Hänchen shift.]

la polarisation TE et la polarisation TM. Cette différence est donnée par :

$$
\phi=\left|\varphi_{\mathrm{TE}}-\varphi_{\mathrm{TM}}\right|
$$

où $\varphi_{\mathrm{TE}}$ et $\varphi_{\mathrm{TM}}$ sont les phases introduites lors de la réflexion totale de la lumière sur l'objet et s'expriment sous la forme [8]:

$$
\begin{aligned}
& \varphi_{\mathrm{TE}}=2 \operatorname{Arctg}\left\{\frac{n \sqrt{n^{2} \sin ^{2} \theta-1}}{\cos \theta}\right\} \\
& \varphi_{\mathrm{TM}}=2 \operatorname{Arctg}\left\{\frac{\sqrt{n^{2} \sin ^{2} \theta-1}}{n \cos \theta}\right\}
\end{aligned}
$$

pour $\theta=45$ degrés et $n=1.515$, la différence de phase vaut 39 degrés environ ce qui correspond à un déplacement de $69 \mathrm{~nm}$ du miroir.

La figure 10 est obtenue en travaillant avec une polarisation quelconque entre TE et TM. A la détection, tout se passe comme si le champ électrique de l'onde électromagnétique incidente était projeté dans la base propre de polarisation (TE-TM). On retrouve alors le fonctionnement classique de l'interféromètre comme filtre de polarisation. 




Fig. 10. - Pour une polarisation intermédiaire entre TE et TM, la position du miroir permet de filtrer telle ou telle composante de la polarisation. Linterféromètre est utilisé comme filtre de polarisation.

[Plotted intensity curves versus the mirror position in the case of a mixture of TE and TM polarization. The cavity can be also compared with a polarization filter.]

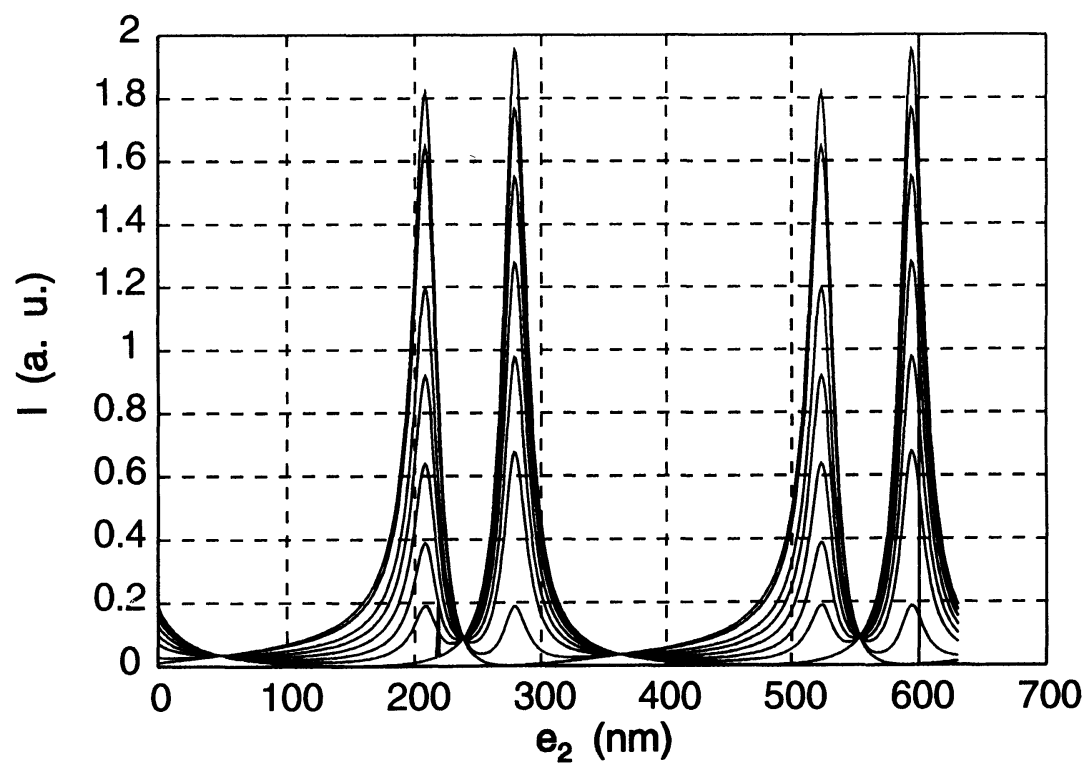

Fig. 11. - La polarisation de l'onde incidente varie de TE à TM en passant par des cas intermédiaires. Les variations de l'intensité détectée font apparaître l'existence de deux points particuliers par période, lieux d'intersection de toutes ces courbes. En ces points, l'intensité lumineuse détectée est indépendante de la polarisation incidente.

[While the incident wave polarization is moving from TE to TM the intensity variations show two particular points per period (intersection points). In such particular configurations the detected light intensity is independent of the incident polarization.] 
La figure 11 représente les variations de l'intensité lumineuse en fonction de la position du miroir pour plusieurs polarisations. On remarque au cours d'une période, l'existence de deux points particuliers, lieux d'intersection de toutes les courbes. Ceux-ci correspondent à deux configurations différentes du miroir et de la lame semi-transparente, pour lesquelles l'intensité lumineuse détectée au-dessus du dioptre est constante.

Ces phénomènes, dus à la polarisation, peuvent être également mis en évidence en considérant une sonde plate. La seule différence réside dans le fait que le couplage va introduire un glissement et un élargissement du pic de résonance. C'est cet effet que l'on doit exploiter pour pouvoir caractériser la qualité d'une sonde. En effet, plus la sonde est étendue plus le couplage est fort et plus le pic de résonance est large. Au contraire, dans le cas d'une sonde très fine, même placée au voisinage immédiat de l'objet, le pic de résonance sera peu perturbé. Un montage expérimental a été imaginé pour contrôler les pointes in situ lors d'une expérience de microscopie en champ proche (Fig. 12).

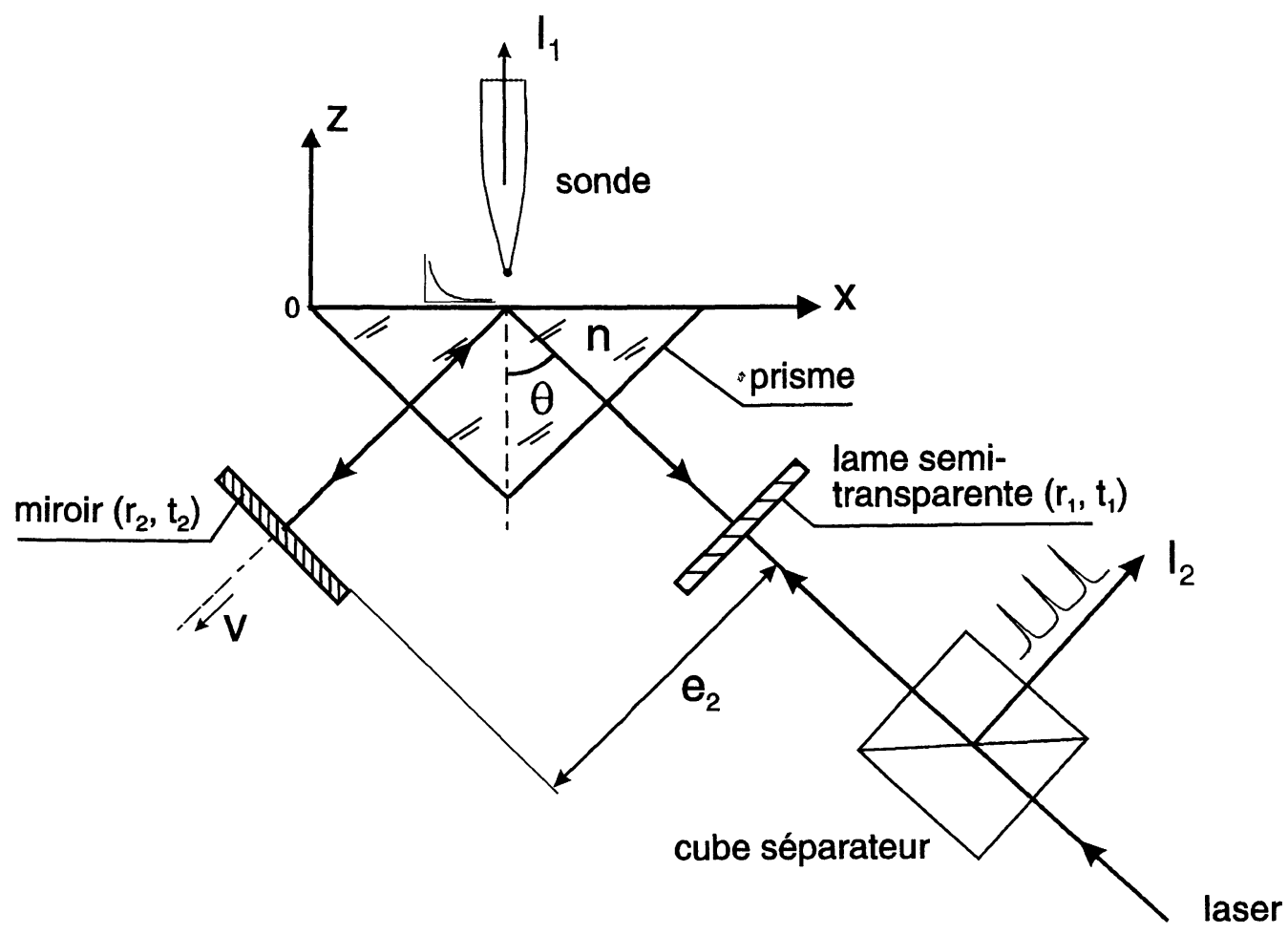

Fig. 12. - Schéma du montage expérimental permettant de contrôler la qualité d'une sonde de microscope optique en champ proche. La lecture de $I_{1}$ nous informe du positionnement de la sonde par rapport à l'objet (exponentielle de Fresnel) alors qu'en déplaçant le miroir suivant la direction $\nu$ avec une amplitude supérieure ou égale à $\frac{\lambda}{2}$ et en détectant $I_{2}$ (donc la finesse), on détecte, in situ, l'effet de couplage entre la sonde et l'objet.

[Experimental set-up allowing the control of the quality of probes used in near field optical microscope. The detection of the intensities $I_{1}$ and $I_{2}$ gives information about the distance probe-object and the coupling probe-object respectively.]

La figure 13 représente les variations du coefficient de finesse calculé comme étant le rapport entre la période des résonances $(\lambda / 2)$ sur la largeur à mi-hauteur du pic en fonction de la distance séparant la sonde de l'objet. Dans le cas d'une sonde très fine (pas de couplage), la finesse est 
indépendante de la position de la sonde, elle est donnée en fonction des coefficients de réflexion de la lame semi-transparente et du miroir $\left(f=\frac{\pi \sqrt{r_{1} r_{2}}}{1-r_{1} r_{2}}\right)$. Dans le cas d'une sonde large (fibre clivée par exemple), la finesse va dépendre du coefficient de réflexion sur la surface de l'objet (réflexion totale) qui dépend lui-même de la distance entre la sonde et l'objet. Dans le cas réel, où la sonde va avoir un comportement mixte et où la diffraction de la lumière par cette dernière doit être prise en compte, la finesse varie en fonction de $z$ tout en restant entre les deux courbes précédentes.

Les courbes de la figure 7 montrent clairement l'effet de couplage sur la position du pic de résonance. On remarque qu'en éloignant la sonde de l'objet deux effets vont cohabiter donnant lieu à des variations non monotones de l'intensité lumineuse détectée. Le premier effet est celui de l'exponentielle décroissante de l'onde évanescente et le deuxième est celui de la condition de résonance qui peut correspondre à telle ou telle position du miroir. Ces deux effets interfèrent constructivement ou destructivement (effet compétitif) conduisant parfois à des décroissances de l'intensité lumineuse plus rapide ou au contraire plus lente qu'une exponentielle classique.

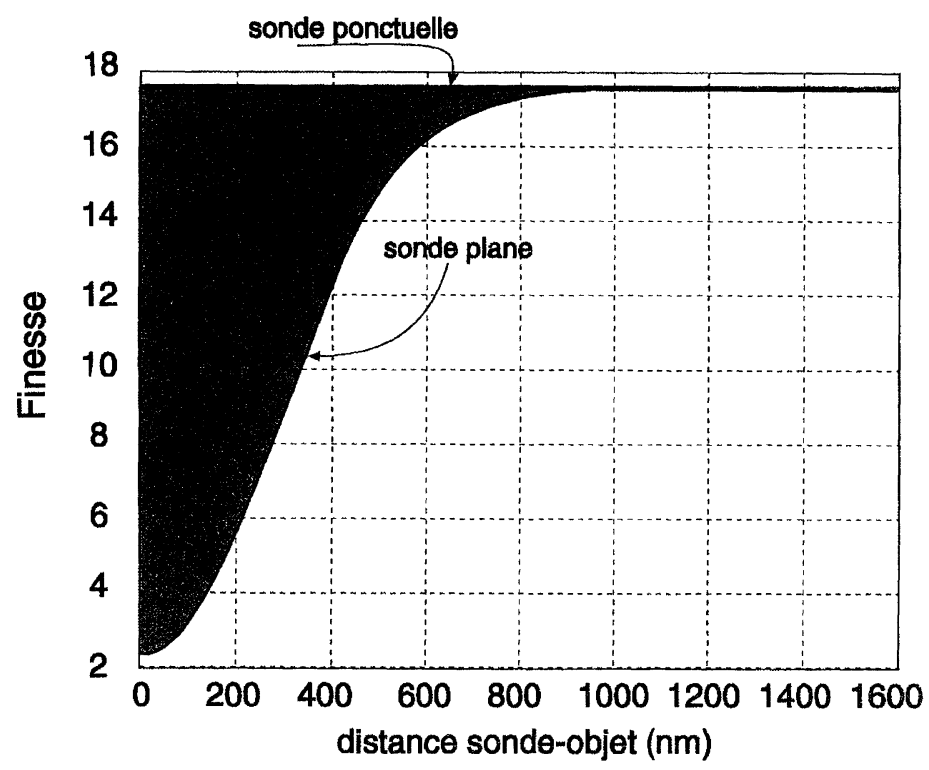

Fig. 13. - Diagramme donnant la finesse de la cavité résonante en fonction de la distance entre la sonde et l'objet. Le cas de la sonde réelle quelconque est très difficilement calculable, mais on peut affirmer que la forme géométrique et l'indice de la sonde vont affecter ce paramètre. La valeur limite supérieure est celle de l'interféromètre en l'absence de sonde. Ce diagramme a été obtenu pour les valeurs suivantes : $n=1.515$, $\lambda=632.8 \mathrm{~nm}, r_{1}=0.86, r_{2}=1$ et indice de la sonde $n_{\mathrm{s}}=1.48$.

[Diagram showing the finesse of the cavity versus probe-object distance. The dashed zone corresponds to all possible cases from punctual probes (upper horizontal line) to flat probes (curve). The simulation parameters are: $n=1.515, \lambda=632.8 \mathrm{~nm}, r_{1}=0.86, r_{2}=1$ and optical index of the probe $n_{\mathrm{s}}=1.48$.]

Un cas particulièrement intéressant peut être évoqué : il consiste à placer la sonde assez loin de l'objet et à déplacer le miroir afin de travailler en régime d'anti-résonance (détection en champ sombre). En gardant la position du miroir fixe, on approche la sonde vers l'objet provoquant ainsi une augmentation brusque de l'intensité lumineuse qui va être surtout due au glissement de la condition d'anti-résonance (la sonde faisant partie de l'interféromètre, sa position va influer sur les conditions de résonance et d'anti-résonance). Cette simulation a pour objet de montrer que l'utilisation d'un tel interféromètre nous permet de s'affranchir de la détection de l'exponentielke 
de Fresnel qui n'apporte en elle-même aucune super-résolution à la microscopie en champ proche optique et qui va se comporter comme un fond continu réduisant le rapport signal sur bruit des images obtenues.

\section{Résultats expérimentaux.}

La première série d'enregistrement a été effectuée en balayant la surface du prisme avec la sonde. La figure 14a montre la distribution d'intensité lumineuse sur la surface du prisme dans le cas classique d'un microscope en champ proche sans interféromètre (sans miroir) alors que la figure 14b montre le même site dans le cas où le microscope est dans la cavité résonante. La période des franges est parfaitement en accord avec celle que prédit la théorie.
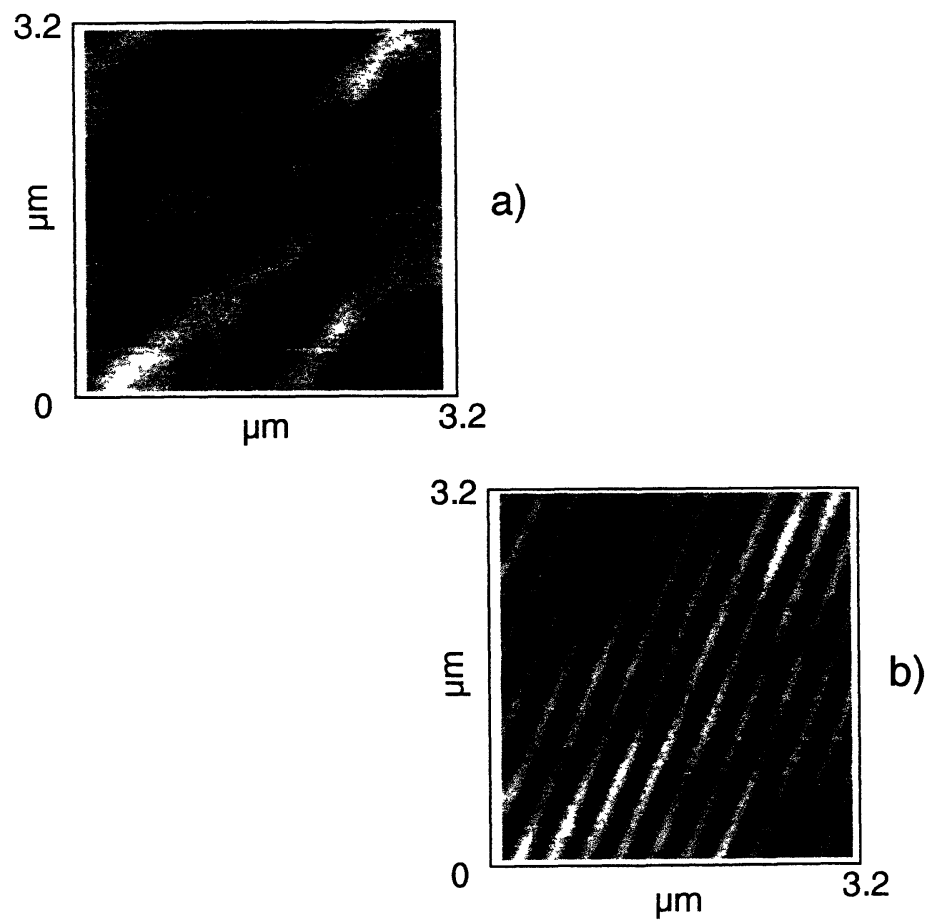

Fig. 14. - Mesures expérimentales de l'intensité lumineuse détectée à la surface du prisme. a) cas classique de microscopie en champ proche (pas de miroir), b) cas étudié avec la cavité interférométrique. Le système de franges est sinusoïdal de période $294 \mathrm{~nm}$ (à comparer avec la Fig. 5).

[Experimental recordings of the detected intensity over the surface of the prism. a) classical set-up (without mirror), b) interferometric set-up. The period of the sinusoidal fringes is $294 \mathrm{~nm}$ (to be compared with Fig. 5).]

En fixant maintenant la position de la sonde, on trace les variations de l'intensité lumineuse détectée en fonction de la position du miroir pour différentes polarisations. Les courbes obtenues sur les figures 15 sont à comparer avec les résultats théoriques des figures 9 et 11 . Sur la figure 16 sont portées les variations $I=f\left(e_{2}\right)$ pour plusieurs polarisations allant de TE à TM, la correspondance avec le résultat de la figure 11 est visible. 




a)
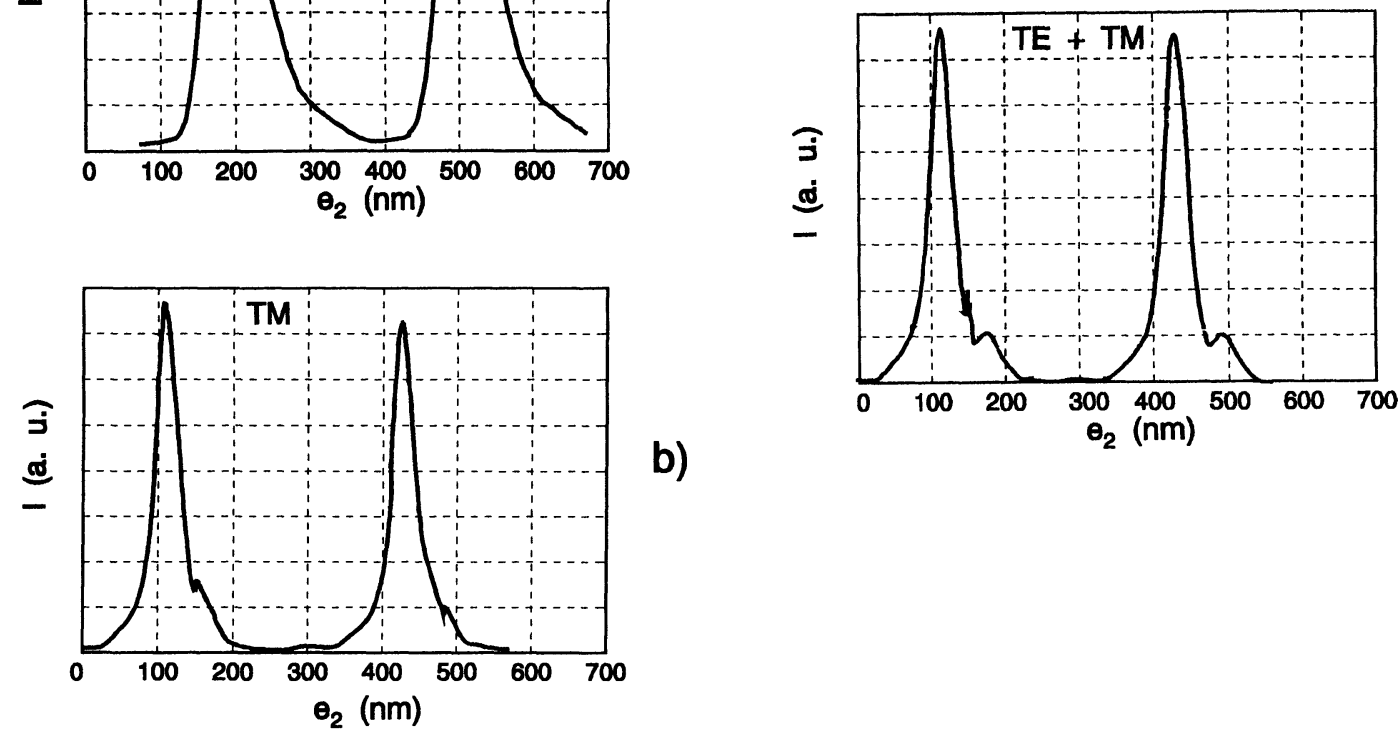

c)

b)

Fig. 15. - Enregistrements des variations de l'intensité lumineuse en fonction de la position du miroir. a) polarisation TE, b) polarisation TM et c) polarisation quelconque (à comparer avec les Figs. 9 et 10).

[Detected intensity variations versus the mirror position. a) TE polarization, b) TM polarization and c) one intermediate plarization (to be compared with Figs. 9 and 10).]

Ces résultats démontrent la capacité future de ce montage à effectuer des spectroscopies locales à haute résolution par simple transposition dans le champ proche de la spectroscopie Fabry-Pérot [9].

On peut aussi remarquer sur la figure 17 l'accord avec la théorie lorsque l'intensité lumineuse varie en fonction de la distance entre la sonde et l'objet.

La figure 18 montre la décroissance exponentielle de l'intensité lumineuse dans un STOM classique (a) comparée à celle obtenue dans le cas d'une détection en champ sombre (b). Une réduction d'un facteur 3.2 de la profondeur de pénétration a pu être mise en évidence. Il s'agit là probablement d'une méthode permettant d'augmenter la résolution latérale et verticale d'un tel microscope [10].

\section{Conclusion et perspectives.}

La microscopie en champ proche est un outil puissant d'analyse des champs d'interférence à caractère non radiatif. Nous avons montré que l'on peut ainsi explorer avec précision le champ lumineux à l'intérieur d'un Fabry-Pérot. Linformation résultante permet de fournir un critère de qualité de la pointe exploratrice (la sonde tunnel).

Bien que le modèle théorique utilisé soit relativement simple, nous avons montré le bon accord entre les données simulées et les données expérimentales en fonction des paramètres physiques tels que la polarisation et la distance d'analyse. 




Fig. 16. - Enregistrement des variations de l'intensité lumineuse en fonction de la position du miroir pour plusieurs polarisations comprises entre TE et TM. On constate l'existence des deux points d'intersection (à comparer avec la Fig. 11).

[Detected intensity variations versus the mirror position for several polarizations (from TE to TM). We point out intersection points (to be compared with Fig. 11).]

Ces résultats ont mis en évidence l'existence d'une configuration particulière pour laquelle le Fabry-Pérot se comporte comme une cavité anti-résonante permettant d'annuler localement le champ évanescent. Cette condition permet de réduire la profondeur de pénétration de l'onde évanescente, elle sera très prochainement utilisée en imagerie champ proche à fond sombre.

Sur le plan théorique, une méthode plus complète (perturbative à l'ordre deux) que celle déjà développée dans notre laboratoire [11] est en cours d'étude. Elle permettra le calcul du coefficient de réflexion spéculaire sur la surface de l'objet en tenant compte de la diffraction de la lumière par une sonde de forme géométrique quelconque et ouvrira le champ à une étude complète du coefficient de finesse de la cavité interférométrique en fonction de la taille et de la forme de la sonde. 

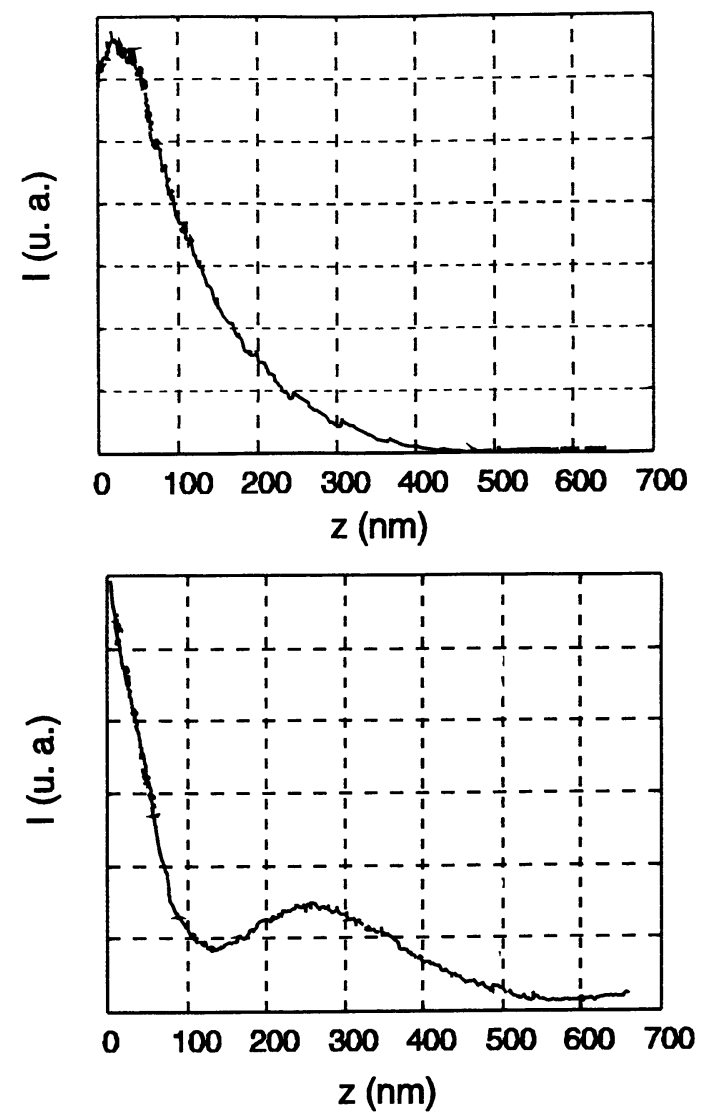

Fig. 17. - Enregistrement des variations de l'intensité lumineuse en fonction de la distance sonde-objet pour deux positions différentes du miroir (à comparer avec les Figs. $7 \mathrm{~b}$ et $7 \mathrm{~d}$ ).

[Detected intensity variations versus the probe-object distance for two different positions of the mirror (to be compared with Figs. $7 b$ and $7 d)$.]



Fig. 18. - Enregistrement de l'intensité lumineuse en fonction de la distance sonde-objet. a) cas du STOM classique, b) détection en champ sombre (cavité travaillant en régime anti-résonant).

[Detected intensity variations versus the probe-object distance. a) classical STOM, b) dark field detection (anti-resonant mode of the cavity).] 


\section{Bibliographie}

[1] Nassenstein H., "Holographie und interferenzversuche mit inhomogenen oberflächenwellen”, Phys. Lett. 28A (1968) 249-251.

[2] Bryngdahl O., "Holography with evanescent waves", JOSA 59 (1969) 1645-1650.

[3] Meixner A., Bopp M. et Tarrach G., "Direct Measurement of Standing Evanescent Waves with a Photon Scanning Tunneling Microscope", à paraître dans Appl. Opt. 33 (1994).

[4] Mertz J., Hipp M., Mlynek J. et Marti O., "Optical Near-Field Imaging with a Semiconductor Probe Tip”, à paraître dans Appl. Phys. Lett.

[5] Courjon D., Bainier C. et Baida F., "Seeing inside a Fabry-Pérot resonator by means of a scanning tunneling optical microscope", Opt. Comm. 110 (1994) 7-12.

[6] Courjon D., Sarayeddine K. et Spajer M., "Scanning tunneling optical microscopy", Opt. Comm. 71 (1989) 23-28.

[7] Vigoureux J.M., "Polynomial formulation of reflection and transmission by stratified planar structures", JOSA A 11 (1991) 1697-1701.

[8] Manallah A., "Etude du décalage longitudinal et du décalage transversal d'un faisceau lumineux en réflexion totale : mesure par détection hétérodyne", Thèse Sciences pour l'Ingénieur (Université de Franche-Comté, Besançon, 1988).

[9] Born M. et Wolf E., Principles of Optics (Pergamon Press, 1959) 332.

[10] Pohl D.W., Courjon D., Bainier C., Dereux A. et Heinzelmann H., "Optical tunneling through adjustable liquid metal gap”, Proceedings NATO 242 (1992) 46-51.

[11] Van Labeke D. et Barchiesi D., "Scanning Tunneling Optical Microscopy: a theoretical macroscopic approach", JOSA A 5 (1992) 732-739. 